Revista Colombiana de Obstetricia y Ginecología Vol. 55 No.4 • 2004 • (293-299)

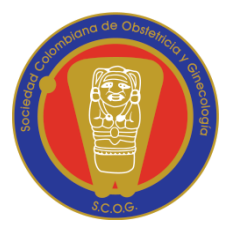

InVESTIGACión Original

\title{
IMPORTANCIA DE LA RESPUESTA A LA INDUCCIÓN DE OVULACIÓN Y LA EDAD EN EL RESULTADO DE LAS TÉCNICAS DE REPRODUCCIÓN ASISTIDA
}

\author{
IMPORTANCE OF THE RESPONSE TO THE OVUILATION INDUCTION \\ AND THE AGE IN THE RESUILT OF THE ASSISTED REPRODUCTION \\ TECHINIQUIES
}

José Ignacio Madero, M.D.*, Claudia López, M.D.**, María Fernanda León, M.D.***, Jair Ruiz, M.D.****, Carlos Gutiérrez, M.D.*****, Óscar Guerra, M.D.******, Mábel Avila, M.D.*******, Magally Escobar, M.D.********, Jesús A. Ruiz, M.D.**********

Recibido: septiembre 16/04 - Revisado: octubre 14/04 - Aceptado: noviembre 22/04

\section{RESUMEN}

Objetivo: este estudio pretendió analizar la eficacia de las técnicas de reproducción asistida en un grupo de pacientes con una respuesta disminuida a la estimulación ovárica (tres folículos o menos al finalizar la estimulación ovárica) y correlacionarla con la edad.

Materiales y métodos: se describen 966 casos de pacientes sometidas a fertilización in vitro o inyección intracitoplasmática de espermatozoides, de las cuales 100 tuvieron una respuesta pobre .

Resultados: el grupo de las «pobres respondedoras» en un $80 \%$ de los casos correspondió a mujeres mayores de 35 años, siendo el 46\% mayor de 40. El promedio de embriones transferidos fue 1,59 para el grupo de estudio y 3,47 para el grupo

Medifertil, Programa de Medicina Reproductiva, Pontificia Universidad Javeriana. Medifertil. Calle 127 No 28-75. Bogotá, Colombia. Correo electrónico: madero@medifertil.com

** Medifertil, Programa de Medicina Reproductiva, Pontificia Universidad Javeriana

*** Medifertil, Programa de Medicina Reproductiva, Pontificia Universidad Javeriana

***** Departamento de Ginecología y Obstetricia. Hospital San Ignacio, Pontificia Universidad Javeriana

***** Instituto de Genética Human. Pontificia Universidad Javeriana.

******* Departamento de Ginecología y Obstetricia. Hospital San Ignacio, Pontificia Universidad Javeriana

********* Medifertil, Programa de Medicina Reproductiva, Pontificia Universidad Javeriana

********* Medifertil, Programa de Medicina Reproductiva, Pontificia Universidad Javeriana

********** Medifertil, Programa de Medicina Reproductiva, Pontificia Universidad Javeriana control, con una tasa de implantación de 11,34 y 12,34\% respectivamente. La tasa de embarazo fue menor en el grupo de las «pobres respondedoras» (16,39\%) comparado con el grupo control (29,62\%).

Conclusiones: los resultados sugieren que las pacientes con una respuesta pobre a la inducción de ovulación (tres folículos o menos) tienen mayor riesgo de un resultado adverso al someterse a técnicas de reproducción asistida por la poca producción de ovocitos, lo cual limita la posibilidad de seleccionar los mejores embriones para transferir y aumenta la probabilidad de obtener embriones con anomalías cromosómicas.

Palabras clave: inducción de ovulación, «pobre respondedora», reserva ovárica, fertilización in vitro, ICSI, FSH, edad.

\section{SUMMARY}

Objective: the aim of the present study is to do an analysis about the efficacy of assisted reproduction techniques in a group of women with diminished response to ovary stimulation (three or less follicles at the end of stimulation process) and its correlation with age.

Materials: we perform an analysis of 966 cases of patients who undergo to in vitro fertilization or intracytoplasmatic spermatozoids injection, 100 of these cases had poor response. 
Results: $80 \%$ of women in the group with poor response were 35 years or older, with $46 \%$ older than 40 years old. The mean of embryo transfer was 1.59 for the study group and 3.47 for the control group with an implantation rate of $11.34 \%$ and $12.34 \%$ respectively. The pregnancy rate was less within the poor response women group than (16.39\%) than the control group (29.62\%).

Conclusions: our results suggest that women with poor response to ovulation induction, it means three or less follicles have higher risk of adverse results when they go through assisted reproduction techniques probably because of the presence of low oocytes counts limits the possibility to select the best embryos to transfer and increase the chance of obtaining embryos with abnormal chromosomal constitution.

Key words: ovulation induction, poor responder, ovarian reserve, in vitro fertilization, ICSI, FSH, age.

\section{INTRODUCCIÓN}

La respuesta pobre a la inducción de la ovulación en los programas de reproducción asistida sigue siendo un reto para los mismos, no obstante haberse diseñado múltiples protocolos para su solución. ${ }^{1,2}$ El concepto de reserva ovárica se utiliza para describir la calidad y cantidad de la población de ovocitos y su relación con el resultado reproductivo, y ha adquirido mayor importancia en las mujeres que serán sometidas a técnicas de reproducción asistida (TRA). Se han desarrollado muchas investigaciones tratando de obtener métodos para predecir el posible resultado de los diferentes tratamientos, incluyendo la determinación de los niveles séricos basales de FSH, LH y estradiol, estudio del volumen ovárico por ecografía, pruebas dinámicas de estimulación con análogo de GnRH, determinación de los niveles de hormona antimulleriana y niveles de inhibina $\mathrm{B}$, entre otros. ${ }^{3,4}$ Una reserva ovárica disminuida da como resultado una respuesta subóptima a los diversos y diferentes esquemas de la inducción de la ovulación, con pocos ovocitos y por consiguiente pocos embriones para transferir, lo cual se traduce en una tasa de embarazo muy baja. ${ }^{5-7}$ A la población de pacientes con este tipo de respuesta se les llama «malas o pobres respondedoras».

\section{Causas de respuesta pobre}

- Edad.

- Genéticas.

- Falla ovárica oculta.

- Autoinmune.

- Infección: ooforitis viral.

- Iatrogénicas: cirugías pélvicas, radioterapia, quimioterapia.

- Idiopáticas: tóxicos del medio ambiente(?). ${ }^{8}$

En lo que estamos de acuerdo es en la disminución del potencial reproductivo con la edad y si a esto le agregamos que en la actualidad la mujer por diversas causas ha pospuesto su maternidad, este grupo de «pobres respondedoras» pasa a ser importante y numeroso. ${ }^{9}$

Los cambios de la capacidad reproductiva están relacionados directamente con la edad de la mujer y se caracterizan por disminución en la cantidad de folículos ováricos, una ovogénesis anormal y cambios en la función endocrina.

En la etapa embrionaria aparece un pico en el número de ovocitos (quinto mes), el cual disminuye progresivamente hasta alcanzar en la etapa de la menarquia un número aproximado de 300.000. Esta pérdida de folículos continúa a través de la vida reproductiva y se acelera unos diez años antes de la aparición de la menopausia. Para esa época los ovocitos han caído a unos $1.000 .{ }^{10,11}$ Así mismo, la edad de aparición de la menopausia también es variable, en nuestro medio es a los 50 años de edad, ${ }^{12}$ pero con variaciones individuales que pueden ir de los 40 a los 60 años. Pero si el $10 \%$ de las mujeres tiene la menopausia a los 46 años de edad, quiere decir que su fertilidad está comprometida desde la mitad de los 30. De otro lado, los ovocitos que potencialmente se desarrollarán durante toda la vida reproductiva ya están presentes desde la menarquia, lo cual hace que a esa edad los ovocitos sean «viejos». 
Identificar adecuadamente este grupo de pacientes tiene un papel determinante en los resultados de los TRA, ya que requieren de un manejo especializado para tratar de optimizar el número y la calidad de los ovocitos recuperados y así incrementar las tasas de implantación y embarazo que se encuentran disminuidas cuando se comparan con los grupos controles normales, aun con esquemas de inducción de ovulación considerados adecuados. ${ }^{13,14}$

El objetivo del presente estudio retrospectivo fue describir los resultados de las TRA de un grupo de pacientes con respuesta disminuida a la inducción de ovulación, definida por el número de folículos observados al final de la estimulación ovárica (tres folículos o menos) y en un grupo de pacientes con respuesta normal.

\section{MATERIALES Y MÉTODOS}

Diseño: estudio descriptivo.

Población y lugar: se analizaron los resultados de 966 ciclos de parejas sometidas a TRA con fertilización in vitro (FIV) e inyección intracitoplasmática de un espermatozoide (ICSI), que fueron realizadas durante el periodo comprendido entre enero de 1998 hasta el 31 de junio de 2003, en Medifertil, Programa de Medicina Reproductiva en Bogotá, Colombia. La población fue dividida en dos grupos de estudio; el primero conformado por pacientes con baja respuesta a la inducción de la ovulación, con tres folículos o menos monitorizados por ecografía transvaginal durante la estimulación ovárica, quienes fueron comparadas con un segundo grupo (control) de mujeres que tuvieron más de tres folículos monitorizados durante la inducción de la ovulación.

\section{Criterios de inclusión - exclusión}

Los criterios de inclusión fueron: ciclos menstruales regulares (26-35 días), presencia de ambos ovarios, sin evidencia de alteraciones endocrinas ni de hidrosálpinx, y finalmente la firma del consentimiento informado.
En los criterios de exclusión para el análisis de datos, se excluyeron 38 ciclos en los cuales no hubo recuperación de ovocitos luego de la aspiración.

\section{Protocolos de estimulación ovárica}

Luego de realizado el estudio de la pareja para ser sometida a TRA, se eligió uno de los siguientes esquemas de inducción de ovulación: desensibilización hipofisaria con análogo de GnRH en protocolo largo, utilizando 0,5 mg subcutáneos al día; se empezó en el día 20 del ciclo menstrual previo o en protocolo corto a partir del día primero del ciclo menstrual, luego en el día tres del ciclo se inició Folitropin Alfa (Gonal F) o FSH urinaria (Follitrin) o HMG Massone en dosis de 150 UI al día.

Otro grupo de pacientes recibió $100 \mathrm{mg}$ de citrato de clomifeno del día dos al seis, combinado con 150 UI de HMG del día dos al ocho o un esquema de solo FSH del día dos al ocho en dosis fijas de 150 UI. Finalmente, un grupo recibió manejo con FSH más antagonista de GnRH, el cual se inició cuando por ecografía transvaginal el promedio de los folículos estaba en $14 \mathrm{~mm}$. La estimulación ovárica se monitorizó mediante ecografía transvaginal y niveles de estradiol. Las dosis de gonadotropinas, si era necesario, se reajustaban con base en la respuesta ovárica. Cuando los folículos ováricos dominantes se hallaban en promedio en $18 \mathrm{~mm}$ y el endometrio en mínimo $8 \mathrm{~mm}$, trilaminar, se procedía a colocar la gonadotropina coriónica humana (Profasi; Serono o Gonacor; Massone) para realizar la aspiración folicular 36 horas más tarde. ${ }^{15}$

Se cancelaron los ciclos en los cuales no hubo un crecimiento folicular, ya que este es un indicador de mal pronóstico para el ciclo.

\section{Variables para medir}

Se analizaron variables como la edad de la paciente, esquemas de estimulación (variables predictoras), calidad ovocitaria y embrionaria, tasas de embarazo, entre otras (variables resultado). Las pacientes fueron clasificadas según la edad en tres categorías: menores de 35, de 35 a 39 y más de 40 años. 


\section{RESULTADOS}

De los 966 ciclos analizados, 100 pertenecían al grupo de «pobres respondedoras» y 866 correspondieron a pacientes con respuesta adecuada. En 17 de los 100 ciclos de respuesta pobre no hubo recuperación de ovocitos luego de la aspiración y en 21 de los 866 del grupo control, lo cual da un total de 928 ciclos con recuperación de ovocitos.

$\mathrm{Al}$ analizar la edad se observó que el 80\% del total de casos, en el grupo de las mujeres con menos de tres folículos, estuvo constituido por pacientes mayores de 35 años (tabla 1).

Según los esquemas de inducción de ovulación, la combinación de citrato de clomifeno más HMG se halló en el 61\% de los casos de respuesta pobre, contra un 43,6\% del grupo control (tabla 2).

Luego de la recuperación de ovocitos se valoró la calidad ovocitaria, teniendo en cuenta el complejo cúmulo-corona-ovocito (CCO), entre los diferentes grupos analizados. Los resultados se muestran gráfica $\mathbf{1}$.

El porcentaje de ciclos con transferencia fue: $73,5 \%$ para el grupo de pobre respuesta y del

\begin{tabular}{ccc}
\multicolumn{3}{c}{ Tabla 1. Distribución de } \\
pacientes con base en la edad \\
$<$ de 3 folículos & $>$ de 3 folículos \\
& $\mathbf{n}=\mathbf{8 3}(\%)$ & $\mathbf{n}=\mathbf{8 4 5}(\%)$ \\
\hline$<35$ años & 19,27 & 54,67 \\
\hline $35-39$ años & 33,73 & 30,29 \\
\hline$>40$ años & 46,98 & 15,02 \\
\hline
\end{tabular}

Tabla 2. Distribución de pacientes según el esquema de estimulación

\begin{tabular}{lcc} 
& $\begin{array}{c}\text { Menos de } \\
\text { 3 folículos } \\
\text { (\%) }\end{array}$ & $\begin{array}{c}\text { Más de } \\
\text { folículos } \\
\text { (\%) }\end{array}$ \\
\hline CC + HMG & 61 & 43,6 \\
\hline GnRHa + FSH & 18 & 22 \\
\hline GnRHa + HMG & 13,2 & 25,08 \\
\hline GnRHa + FSH + HMG & 0 & 1,65 \\
\hline Solo FSH & 7,2 & 6,15 \\
\hline GnRH Antag + FSH & 0 & 0,59 \\
\hline
\end{tabular}

92,6\% para el grupo control. En cuanto a las tasas de fertilización fueron $72,48 \%$ para el grupo de menos de tres folículos y 70,78\% para el grupo control. La calidad embrionaria se muestra en la gráfica 2. La relación entre la calidad embrionaria y la edad de la paciente se observa en las tablas 3 y 4 .

El promedio de embriones transferidos fue 1,59 para el grupo de estudio y 3,47 para el grupo control, la tasa de implantación fue de 11,34 y $12,34 \%$ respectivamente y la tasa de embarazo por transferencia 16,39 y $29,62 \%$ para el grupo control. Al relacionar las tasas de embarazo por grupos de edad se obtuvieron los resultados mostrados en la tabla 5.

La tasa de aborto en el grupo de pacientes con una respuesta de menos de tres folículos fue del $30 \%$ y del $12,93 \%$ en el grupo control.

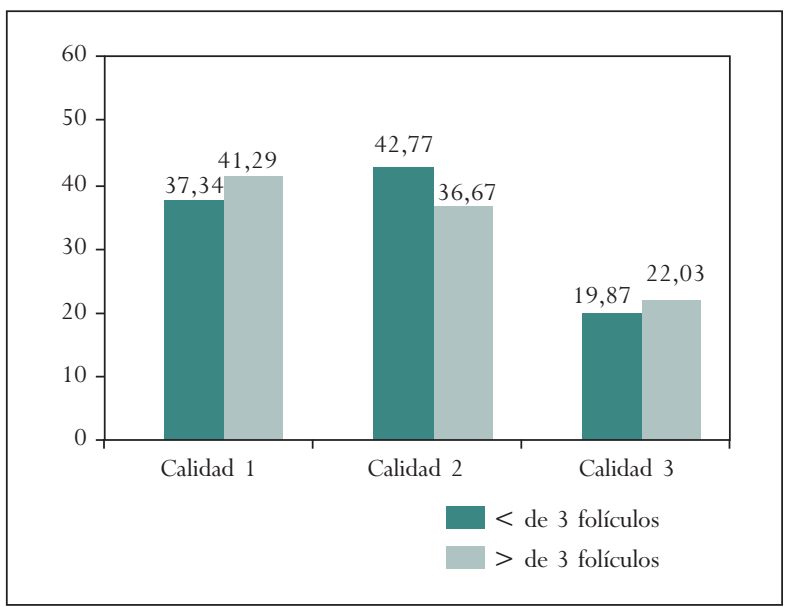

Gráfica 1. Calidad ovocitaria según complejo CCO

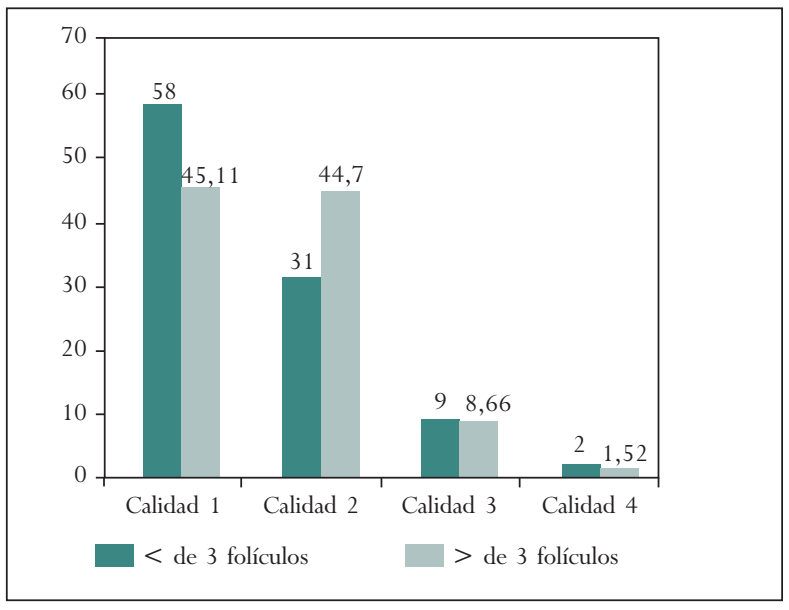

Gráfica 2. Calidad embrionaria 


\section{DISCUSIÓN}

La definición de «pobre respondedora» varía en opinión de diferentes autores, algunos la definen con base en la calidad de los ovocitos obtenidos en la aspiración, ${ }^{16,17}$ otros según los niveles elevados de la FSH en la fase folicular temprana (día 3), los cuales se correlacionan con una tasa de embarazo menor y una tasa de cancelación de ciclos aumentada; ${ }^{18,19}$ los niveles elevados de estradiol en fase folicular temprana y durante el inicio de la estimulación así como el día de la $\mathrm{hCG}^{20}$ son para otros autores predictores de respuesta pobre. Lo anterior muestra una falta de uniformidad en cuanto a la definición de «pobre respondedora». Sin embargo, no hay duda que este grupo de pacientes finalmente tiene un número menor de ovocitos y de embriones para transferir. En el presente estudio, el criterio para definir la «pobre respondedora» fue el número de folículos observados al final de la estimulación ovárica, tres o menos, lo cual es importante para futuras comparaciones.

Tabla 3. Calidad embrionaria según la edad en pacientes de menos de 3 folículos

\begin{tabular}{lcccc} 
& $\begin{array}{c}\text { CAL 1 } \\
(\%)\end{array}$ & $\begin{array}{c}\text { CAL 2 } \\
(\%)\end{array}$ & $\begin{array}{c}\text { CAL 3 } \\
(\%)\end{array}$ & $\begin{array}{c}\text { CAL 4 } \\
(\%)\end{array}$ \\
\hline$<35$ años & 64,28 & 21,42 & 7,14 & 7,14 \\
\hline $35-39$ años & 62,50 & 27.50 & 7,50 & 2,50 \\
\hline$>39$ años & 52,17 & 36,95 & 10,86 & 0 \\
\hline
\end{tabular}

Tabla 4. Calidad embrionaria según la edad en pacientes de más de 3 folículos

\begin{tabular}{cccc}
$\begin{array}{c}\text { CAL 1 } \\
\text { (\%) }\end{array}$ & $\begin{array}{c}\text { CAL 2 } \\
\text { (\%) }\end{array}$ & $\begin{array}{c}\text { CAL 3 } \\
\text { (\%) }\end{array}$ & $\begin{array}{c}\text { CAL 4 } \\
\text { (\%) }\end{array}$ \\
\hline 44,31 & 46,03 & 7,97 & 1,66 \\
\hline 43,91 & 43,80 & 10,86 & 1,41 \\
\hline 51,95 & 40,20 & 6,78 & 1,04 \\
\hline
\end{tabular}

Tabla 5. Tasa de embarazo por edad

$$
<3>3
$$

\begin{tabular}{ccc} 
& folículos (\%) & folículos (\%) \\
\hline$<35$ años & 6,25 & 29,22 \\
\hline $35-39$ a. & 17,8 & 29,68 \\
\hline$>40$ años & 10,2 & 16,5 \\
\hline
\end{tabular}

$\mathrm{Al}$ analizar los resultados de este gran número de pacientes, se encontró que la edad materna es uno de los principales factores pronósticos en el resultado de las «pobres respondedoras» sometidas a TRA. En nuestro estudio, 80,7\% de los casos de «pobres respondedoras» correspondió a mujeres mayores de 35 años, del cual el 47\% eran mayores de 40 años. La tasa de transferencia en el grupo de «pobres respondedoras» fue del 73,5 contra un 92,6\% del grupo control. Así mismo, la tasa de embarazo por transferencia de embriones fue mayor en el grupo control al ser comparado con el grupo de estudio; como se mencionó anteriormente en los resultados, la disminución en la tasa de embarazo en este grupo de "pobres respondedoras» puede estar relacionada no solo con la escasa reserva ovárica, sino con la calidad de los ovocitos y embriones no observable a la luz del microscopio. Los ovocitos de las «pobres respondedoras» pueden ser de mala calidad tal vez porque sean los últimos disponibles en el «pool» ovárico; se ha postulado que los ovocitos al inicio así como al final de la vida reproductiva tienen un incremento de riesgo para enfermedades cromosómicas, esto explicaría la presencia de una curva aplanada tipo J en la incidencia de aneuploidías relacionadas con la edad. Ambas, mujeres muy jóvenes y aquellas de 40 o más años están en alto riesgo de conceptus aneuploides. ${ }^{21}$ Otra hipótesis es la no disyunción del ovocito debido a alteraciones en la microcirculación ovárica, la cual se observa en los casos de envejecimiento ovárico. Esta hipótesis puede explicar también el posible incremento de las tasas de aneuploidías en ovocitos de «pobres respondedoras» jóvenes, en quienes la reserva ovárica está disminuida como resultado de procesos adherenciales o a endometriosis. Munne ${ }^{22}$ y Gianaroli ${ }^{23}$ hallaron un incremento de aneuploidías en embriones de «pobres respondedoras» sin relación con la edad. En otro estudio se observó una correlación entre la presencia de cariotipos anormales con la elevación de la FSH en el día 3 del ciclo menstrual. ${ }^{24}$ 
La tasa de aborto en nuestro grupo de pobres respondedoras fue de $30 \%$ vs. $12,9 \%$ en el grupo control, la cual es similar a la hallada por otros autores. ${ }^{25}$ Así mismo se ha planteado que las pacientes con aborto a repetición o pérdida gestacional temprana sin una causa aparente, tienen una mayor incidencia de niveles elevados de FSH que aquellas con una causa conocida aunque los resultados recientes no lo demuestran. ${ }^{26,27}$ También está demostrado que diferencias en las concentraciones o niveles basales de la FSH están correlacionados con una incidencia mayor del Síndrome de Down como se demostró en un estudio de casos y controles realizado por van Montfrans. ${ }^{28}$

Nuestros resultados indican que las pacientes con una respuesta pobre a la inducción de ovulación (tres o menos folículos) tienen un doble riesgo de mostrar un resultado adverso al someterse a TRA por la poca producción de ovocitos, lo cual limitará la posibilidad de seleccionar los mejores embriones para transferir y la probabilidad de obtener ovocitos con mala calidad cromosómica.

\section{REFERENCIAS}

1. Wang PT, Lee RK, Su JT, Hou JW, Lin MH, Hu YM. Cessation of low dose gonadotropin releasing hormone agonist therapy followed by high-dose gonadotropin stimulation yields a favorable ovarian response in poor response in poor responders. J Assist Reprod Genet 2002;19:1-6.

2. Mahutte NG, Arici A. Poor responders: does the protocol make a difference? Curr Opin Obstet Gynecol 2002;14:275-81.

3. Loverro G, Nappi L, Mei L, Giacomoantonio L, Carriero C, Tartagni M. Evaluation of functional ovarian reserve in 60 patients. Reprod Biomed Online 2003;7:200-4.

4. Fawzy M, Lambert A, Harrison RF, Knight PG, Groome N, Hennelly B, et al. Day 5 inhibin B levels in a treatment cycle are predictive of IVF outcome. Hum Reprod 2002;17:1535-43.

5. Madero JI, López C, Chacón P, et al. Resultados de técnicas de reproducción asistida en pacientes con tres folículos o menos. Medicina Reproductiva 2001;1:79.
6. Tan SL, Royston P, Campbell S, Jacobs HS, Betts J, Mason B, et al. Cumulative conception and livebirth rates after in-vitro fertilization. Lancet 1992;339:1390-4.

7. Fanchin R, de Ziegler D, Olivennes F, Taieb J, Dzik A, Frydman R. Exogenous follicle stimulating hormone ovarian reserve test (EFORT): a simple and reliable screening test for detecting «poor responders» in invitro fertilization. Hum Reprod 1994;9:1607-11.

8. Conway G, Christin-Maitre S. Premature ovarian failure. En: Fauser BCJM (Editor in chief). Reproductive medicine. Molecular, cellular and genetic fundamentals. London: Parthenon publishing group; 2002. p. 587-600.

9. Te Velde ER, Pearson PL. The variability of female reproductive ageing. Hum Reprod Update 2002;8:141-54.

10. Faddy MJ, Gosden RG, Gougeon A, Richardson SJ, Nelson JF. Accelerated disappearance of ovarian follicles in mid-life: implications for forecasting menopause. Hum Reprod 1992;7:1342-6.

11. Faddy MJ, Gosden RG. A model confirming the decline in follicle numbers to the age of the age of menopause in women. Hum Reprod 1996;11:1484-6.

12. Onatra W, Sánchez J, Acuña G, Arango G, Barón G, De la Cruz J, et al. Epidemiología de la menopausia en Colombia: estudio cooperativo. Rev Colomb Obstet Ginecol 1994;45:20-8.

13. Kuspesic A, Kurjak A. Predictors of IVF outcome by three-dimensional ultrasound. Hum Reprod 2002;17:950-5.

14. Scott RT, Ophsal MS, Leonardi MR, Neal GS, Illions EH, Navot D. Life table analysis of pregnancy rates in a general infertility population relative to ovarian reserve and patients age. Hum Reprod 1995;10:1706-10.

15. Madero JI, Neira P, Gaitán P, Sanz E, Avila LM, Escobar M, Ruiz JA. Protocolo previo al inicio de técnicas de reproducción asistida. Rev Colomb Obstet Ginecol 1999;50:95-100.

16. Lashen H, Ledger W, López-Bernal, Barlow D. Poor responders to ovulation induction: is proceeding to in vitro fertilization worthwhile. Hum Reprod 1999;14:964-9.

17. Land JA, Yarmolinskaya MI, Dumoulin JC, Evers JL. High-dose human gonadotropin stimulation in poor responders does not improve in vitro fertilization outcome. Fertil Steril 1996;65:961-5. 
18. Silber SJ, Nagy ZP, Devroey P, Camus M, Van Steirteghem AC. The effect of female age and ovarian reserve on pregnant rate in male infertility: treatment of azoospermia with sperm retrieval and intracytoplasmic sperm injection. Hum Reprod 1997;12:2693-700.

19. Scheffer GJ, Broekmans FJ, Dorland M, Habbema JD, Looman CW, Te Velde ER. Antral follicle counts by transvaginal ultrasonography are related to age in women with proven natural fertility. Fertil Steril 1999;72:845-51.

20. Surrey ES, Bower J, Hilla DM, Ramsey J, Surrey MW. Clinical and endocrine effects of a microdose GnRH agonist flare regimen administered to poor responders who are undergoing in vitro fertilization. Fertil Steril 1998;69:419-24.

21. Gaulden ME. A model that explains the varying frequency of aneuploid children with maternal age (J-shaped curve) as well as aneuploidy of paternal origin. Prog Clin Biol Res 1989;318:253-7.

22. Munne S, Rozenwaks Z, Grifo J, Cohen J. Diagnosis of mayor chromosome aneuploidies in human preimplantation embryos. Hum Reprod 1993;8:2185-91.

23. Gianaroli L, Magli MC, Ferrraretti AP, Fortini D, Tabanelli C, Gergolet M. Gonadal activity and chromosomal constitution of in vitro generated embryos. Mol Cell Endocrinol 2000;161:111-6.
24. Nasseri A, Mukherjee T, Grifo J, Noyes N, Krey L, Copperman AB. Elevated day 3 serum follicle stimulating hormone and/or estradiol may predict fetal aneuploidy. Fertil Steril 1999;71:715-8.

25. Ulug U, Ben-Shlomo I, Turan E, Erden HF, Akman MA, Bahceci M. Conception rates following assisted reproduction in poor responders patients: a retrospective study in 300 consecutive cycles. Reprod Biomed Online 2003;6:439-43.

26. van Montfrans JM, van Hooff MH, Huirne JA, Tanahatoe SJ, Sadrezadeh S, Martens F, et al. Basal FSH concentrations as a marker of ovarian ageing are not related to pregnancy outcome in a general population of women over 30 years. Hum Reprod 2004; 19:430-4.

27. Levi AJ, Raynault MF, Bergh PA, Drews MR, Miller BT, Scott RT Jr. Reproductive outcome in patients with diminished ovarian reserve. Fertil Steril 2001;76:666-9.

28. van Montfrans JM, Dorland M, Oosterhuis GJ, van Vugt JM, Rekers-Mombarg LT, Lambalk CB. Increased concentrations of follicle stimulating hormone in mothers of childrens with Down's syndrome. Lancet 1999;353:1853-4. 